Provided for non-commercial research and education use. Not for reproduction, distribution or commercial use.

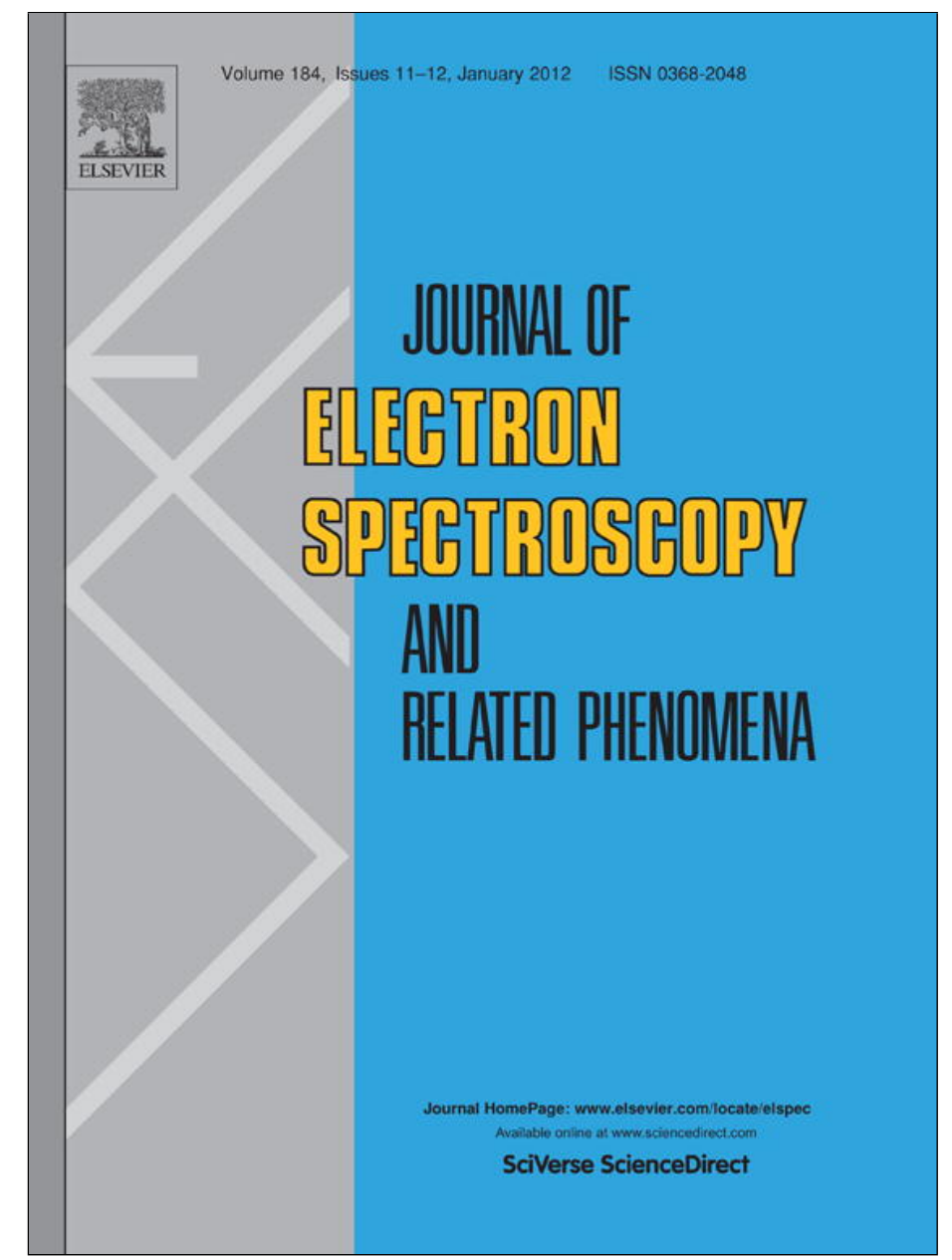

(This is a sample cover image for this issue. The actual cover is not yet available at this time.)

This article appeared in a journal published by Elsevier. The attached copy is furnished to the author for internal non-commercial research and education use, including for instruction at the authors institution and sharing with colleagues.

Other uses, including reproduction and distribution, or selling or licensing copies, or posting to personal, institutional or third party websites are prohibited.

In most cases authors are permitted to post their version of the article (e.g. in Word or Tex form) to their personal website or institutional repository. Authors requiring further information regarding Elsevier's archiving and manuscript policies are encouraged to visit:

http://www.elsevier.com/copyright 


\title{
Charge transfer in the novel donor-acceptor complexes tetra- and hexamethoxypyrene with tetracyanoquinodimethane studied by HAXPES
}

\author{
K. Medjanik ${ }^{\mathrm{a}, *}$, A. Gloskovskii ${ }^{\mathrm{b}}$, D. Kutnyakhov ${ }^{\mathrm{a}}, \mathrm{C}$. Felser ${ }^{\mathrm{b}}$, D. Chercka ${ }^{\mathrm{c}}$, M. Baumgarten $^{\mathrm{c}}, \mathrm{K}$. Müllen ${ }^{\mathrm{c}}$, \\ G. Schönhense ${ }^{a}$ \\ a Institut für Physik, Johannes Gutenberg-Universität, D-55099 Mainz, Germany \\ ${ }^{\mathrm{b}}$ Institut für Anorganische Chemie und Analytische Chemie, Johannes Gutenberg-Universität, D-55099 Mainz, Germany \\ ${ }^{\mathrm{c}}$ Max Planck-Institut für Polymerforschung, Postfach 3148, D-55021 Mainz, Germany
}

\section{A R T I C L E I N F O}

\section{Article history:}

Received 5 December 2011

Received in revised form 6 February 2012

Accepted 6 February 2012

Available online $\mathrm{xxx}$

\section{Keywords:}

Charge transfer salts

TCNQ

Microcrystals

HAXPES

\begin{abstract}
A B S T R A C T
The effect of charge transfer (CT) in complexes of the donors tetra- and hexamethoxyprene (TMP and HMP) with the classical acceptor tetracyanoquinodimethane (TCNQ) was studied using hard X-ray photoemission (HAXPES). Microcrystals of the complex were grown via vapour diffusion from donor-acceptor mixtures. The bulk sensitivity of HAXPES at a photon energy of $6 \mathrm{keV}$ completely eliminates the problem of surface contamination for such delicate organic materials grown from solution. The donor molecules were produced using a novel synthesis route functionalizing polycyclic aromatic hydrocarbons at their periphery. For comparison, spectra were also taken from thin-film samples of the same compounds produced via co-deposition in UHV. Upon complex formation, the oxygen 1s core-level spectra (being a fingerprint of the methoxy-group of the donors) change from the single-line spectrum of pure HMP (TMP) to a spectrum with two distinct lines shifted by $1.4(0.9) \mathrm{eV}$ and $2.6(2.3) \mathrm{eV}$ with respect to the position of the oxygen 1s line of the pure donors. The nitrogen 1s spectra (being a fingerprint of the cyano-group in the acceptor) show two peaks as well with a corresponding shift of $0.9 \mathrm{eV}$ and $2.0 \mathrm{eV}$ in comparison with the leading line of pure TCNQ in opposite direction to the oxygen 1s spectra. These values are substantially larger than shifts in near edge X-ray absorption fine structure (NEXAFS) and ultraviolet photoelectron spectroscopy (UPS) spectra of the same complexes. The changes in the spectra are discussed in terms of the $\mathrm{CT}$ in the complexes. Residues of pure donor and acceptor materials in the microcrystal fractions of the complexes are evident from the presence of non-shifted lines. Peak-area analysis reveals that charge is transferred to a fraction of $60 \%$ of the molecules in the complexes.
\end{abstract}

(c) 2012 Elsevier B.V. All rights reserved.

\section{Introduction}

Novel donor and acceptor molecules based on $\pi$-conjugated ring structures opened a lot of possibilities for the design of novel charge transfer (CT) systems for future applications in development of organic electronics. Modeling new CT systems is one of the main approaches for a designed control of the electronic structure of a special class of materials $[1,2]$. The information depth of hard X-ray photoelectron spectroscopy (HAXPES) is $10-15 \mathrm{~nm}$ at the conditions of the present study. This allows studying such delicate organic systems that do not offer the possibility to clean the surface by standard techniques like ion bombardment. HAXPES is thus suitable for the study of solution-grown crystals and films that were transported through ambient air. Surface contaminations from the solvent, from impurities in the solution or from ambient

\footnotetext{
* Corresponding author. Tel.: +49 61313925412; fax: +49 61313923807.

E-mail address: medyanyk@uni-mainz.de (K. Medjanik).
}

air do not show up significantly in HAXPES spectra. Nevertheless, the state-of-the-art energy resolution of HAXPES provides the full chemical specificity of X-ray photoelectron spectroscopy/electron spectroscopy for chemical analysis (XPS/ESCA), combined with true bulk sensitivity.

The past decade has witnessed tremendous advances in the development of organic conductive molecular and polymeric materials and this field continues to be of great scientific and commercial interest. This field of science flourished on discovering new $\pi$ conjugated materials and by tailoring their electrical conductivity from semiconducting to metallic to the superconducting regime, when doped. Characteristic for such compounds is a highly correlated electronic ground state that can exhibit superconductivity, spin density waves, or charge density waves [2]. The first families of organic conductive and superconductive molecules are based on CT salts, where BEDT-TTF [bis(ethylenedithio)tetrathiafulvalene], $M(d m i t)_{2}$ (dmit=1,3-dithio-2-thione-4,5-dithiolate), DCNQI (N,N'dicyano-p-quinonediimine) perylene, tetrachalcogenafulvalenes and their structurally related analogs are just a few of the most 
commonly studied. Fullerenes constitute another class which also exhibit superconductivity when doped with alkali metals. Research activities are being carried out around the world to produce pure fullerenes (C60, C70, C76, C78, C82, C84, C90, and higher fullerenes) and to prepare their derivatives as well as polymers [3]. Another class of materials of high current interest are the nanographenes [4-7] which can be functionalized at their periphery to design large disc-type donors and acceptors.

The primary source of knowledge of the CT effect was tetrathiafulvalene (TTF)-tetracyanoquinodimethane (TCNQ) and related compounds, which were intensively studied in the middle of the last century. Today novel CT salts based on one of the components of the above mentioned well-known system, give us the possibility to improve our understanding of the CT mechanism and find out new methods to estimate the degree of CT.

We briefly recall some results of previous XPS studies of CT salts from the literature. A study of TTF-TCNQ and related compounds revealed a splitting of about $1.5 \mathrm{eV}$ in the $\mathrm{S} 2 \mathrm{p}(\mathrm{N} 1 \mathrm{~s})$ peak positions between neutral $T T F^{0}\left(T C N Q^{0}\right)$ and ionic $T T F^{+}\left(T C N Q^{-}\right)[1,8]$. This splitting could be used to estimate the amount of charge transfer. The evaluation of the $\mathrm{N} 1 \mathrm{~s}$ spectra for the determination of the CT state of TCNQ turned out not so simple because of a satellite due to shake-up of $\pi$-electrons. Evaluation of the molecular mixed valence of TCNQ was possible for the complexes (BEDT-TTF)TCNQ and ditetramethyltetraselenafulvalene (TMTSF)-TCNQ, where an $\mathrm{N}$ 1s binding-energy difference of $1.6 \mathrm{eV}$ between neutral $T C N Q^{0}$ and ionic $T C N Q^{-}$occurs $[9,10]$. This change is accompanied by a red shift of about $30 \mathrm{~cm}^{-1}$ in the frequency of the $C N$ stretching mode ( $a_{g}$ mode) when going from the neutral TCNQ to the anion.

In the present study, we investigate two new $\mathrm{CT}$ complexes consisting of the classical acceptor 7,7,8,8-tetracyano-pquinodimethane (TCNQ $\mathrm{C}_{12} \mathrm{~N}_{4} \mathrm{H}_{4}$ ) and two newly synthesized donors, i.e. the parent molecule pyrene being substituted at its periphery with four or six methoxy groups, see Fig. 1a. These functional groups transfer charge into the $\pi$-orbitals of the aromatic ring system thereby making 4,5,9,10-tetramethoxypyrene (TMP, $\mathrm{C}_{20} \mathrm{H}_{18} \mathrm{O}_{4}$ ) and 2,4,5,7,9,10-hexamethoxypyrene ( $\mathrm{HMP}, \mathrm{C}_{22} \mathrm{H}_{22} \mathrm{O}_{6}$ ) good electron donors. Indeed, for a ultrahigh vacuum (UHV)-deposited thin film of $T M P-T C N Q$ characteristic changes of the electronic structure occur in ultraviolet photoelectron spectroscopy (UPS) and scanning tunnelling spectroscopy, along with a red-shift of the $C N$ stretching mode measured by infrared (IR) spectroscopy, that give evidence of charge transfer in the co-deposited film [11].

Co-crystals of the complex could be grown from solution and are stable under ambient air conditions. X-ray diffraction revealed a mixed stack geometry [12] which is sketched in Fig. 1b. For $T M P-T C N Q$ the structure did not contain intercalated solvent molecules, whereas for HMP-TCNQ one dimethyl sulfoxide (DMSO) molecule was intercalated per donor-acceptor pair. The symmetric distance $(0.332 \mathrm{~nm}$ ) of TCNQ to a TMP on top and a TMP below reveals that no dimerization occurs. The TCNQ molecules alternatively change from alignment to the upper and lower ring of the pyrene aromatic system. The methoxy groups at positions 2 and 7 are nearly in plane with the pyrene core $\left( \pm 6^{\circ}\right)$, while the four methoxy groups at positions 4,5,9 and 10 are strongly twisted out of the plane by $65-85^{\circ}$. The shortest $\mathrm{C}-\mathrm{C}$ distance is $0.3133 \mathrm{~nm}$ but many short contacts are found in average with $0.32-0.35 \mathrm{~nm}$. Cocrystals of TMP-TCNQ were obtained without intercalated solvent molecules. Here the $\mathrm{C}-\mathrm{C}$ distances are slightly larger with $0.332 \mathrm{~nm}$ as shortest contact of a TMP to both the on-top and bottom TCNQ neighbours. Thus a significant difference between TMP-TCNQ and HMP-TCNQ is evident. In TMP-TCNQ alternating stacks show TMP donor next to donor and TCNQ acceptor next to acceptor, such that layers of donors and layers of acceptors are formed as visible in Fig. 1b.
From the spectroscopic point of view the complexes TMP(HMP)$T C N Q$ bear the advantage that oxygen is only contained in the methoxy group of the donor. Hence, the $01 \mathrm{~s}$ spectra directly probe the functional group that is responsible for the amount of charge transfer. Analogously, nitrogen is only contained in the cyano-group of the acceptor. So, the $\mathrm{N} 1 \mathrm{~s}$ spectra probe the acceptor sites as was exploited in earlier work [8-10,13-15].

\section{Sample preparation and experimental details}

The synthesis of the methoxy-substituted pyrene derivatives 4,5,9,10-tetramethoxypyrene and 2,4,5,7,9,10-hexamethoxypyrene is discussed by Kawano et al. [12]. TCNQ is commercially available (Alfa Aesar GmbH \& Co KG). Microcrystals of TMP-TCNQ and $H M P-T C N Q$ were grown by vapour diffusion of hexane into a dichloromethane solution $\left(5 \mathrm{ml}, 6.2 \times 10^{-3} \mathrm{~mol} / \mathrm{l}\right)$ of the components. Solutions with donor-acceptor mixtures of 1:2, $1: 1$ and 2:1 stoichiometry were prepared. This allows investigating possible differences in crystals growing in donor-rich or acceptor-rich solutions. The components were combined in a glass vial $\left(V=7 \mathrm{~cm}^{3}\right.$. $1.5 \mathrm{~cm}$ diameter) and dissolved under sonication. Vapour diffusion assisted crystallizations were performed in a gas-tight chamber $\left(V=120 \mathrm{~cm}^{3}\right)$, filled with $15 \mathrm{ml}$ hexane. The vial containing the solution was placed inside the chamber which was sealed for 4 days. The crystallites have sizes in the range from several $10 \mu \mathrm{m}$ to several $100 \mu \mathrm{m}$.

Optical microscopy (Fig. 1c and d) revealed mixtures of dark and transparent crystals in the vial. Crystallites of the CT complex appeared dark orange, whereas crystallites of the pure donors $T M P$ or HMP are colorless transparent and TCNQ is transparent with light green color. The different fractions were separated using an own developed micromanipulator under the optical microscope. Fig. 1c and d show fractions of microcrystals before separation and the same fraction after separation of the colored co-crystals, respectively. Although all sufficiently large transparent crystallites had been removed, a residue of very small transparent crystals remained, partly being attached to the larger crystallites of the complex.

Thin films of TMP-TCNQ have been prepared by UHV codeposition using a special type of double-evaporator $[11,16]$. The $T M P-T C N Q$ mixed phase was deposited in very good quality by loading one crucible with a mixture of both molecules. The base pressure before evaporation was about $3 \times 10^{-9}$ mbar. Clean Au films were used as substrates with high electronegativity, providing a good reference for the Fermi energy. About $20 \mathrm{~nm}$ of Au were evaporated onto $\mathrm{Si}(100)$-wafers immediately before deposition of the organic films. The workfunction of the fresh Au films was consistently $5.3 \mathrm{eV}$. This indicates that the Au surface is textured and exhibits $\mathrm{Au}(111)$ facets. The substrate was kept at room temperature; the crucible temperature was $120^{\circ} \mathrm{C}$.

Atomic force microscopy (AFM) images of a TMP-TCNQ film deposited on $\mathrm{Au}$ are shown in Fig. 1e. 3D-islands with lateral sizes in the $1 \mu \mathrm{m}$ range and height in the $100 \mathrm{~nm}$ range appeared on a smooth background. The crystallites tend to form rows with lengths of up to one micrometer. The films look dark orange giving evidence for an optical gap in the visible spectral range. Films of pure $T M P$ and $T C N Q$ look transparent and faint yellow, respectively. We suggest that the complex grows in a Stranski-Krastanov type mode [17] where an initial smooth multilayer covers the whole surface (smooth areas in the AFM images, Fig. 1e) before 3D crystallites are formed. Similar microcrystals with sizes in the sub-micrometer range have been observed for the (BEDT-TTF)-TCNQ mixed phase [18]. After separation, the microcrystal fractions were deposited on carbon tape. Thin films were evaporated in situ immediately before the experiment. 
(a)

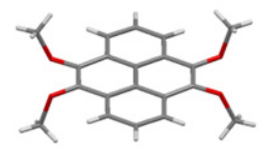

TMP

(b)

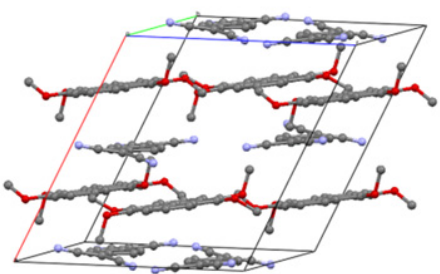

TMP-TCNQ

(c)

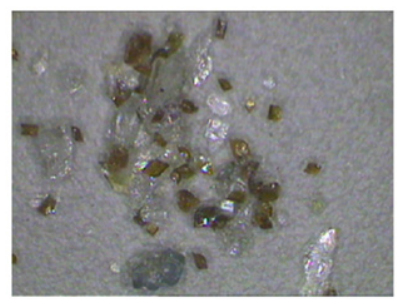

(e)

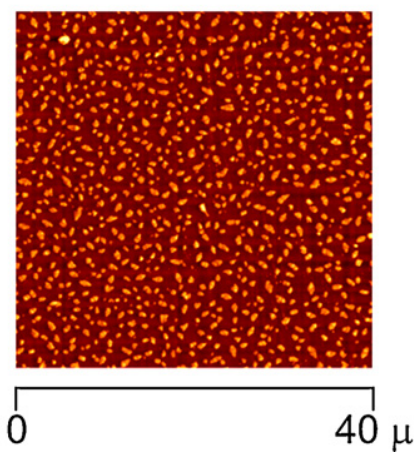

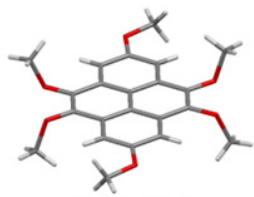

HMP
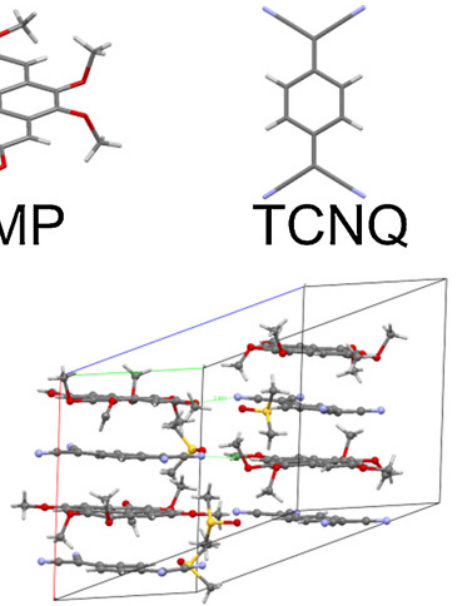

HMP-TCNQ

(d)

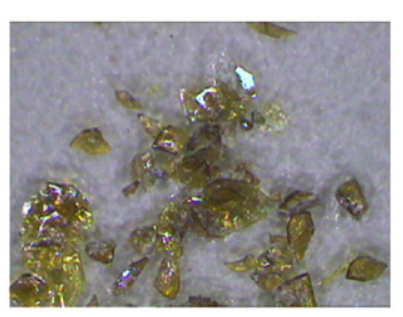

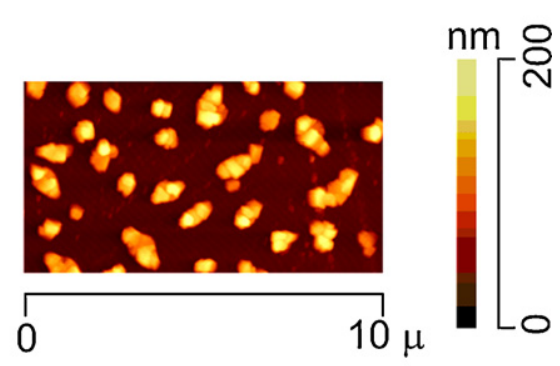

Fig. 1. Structure of the molecules (a) and of the TMP(HMP)-TCNQ complexes as obtained from X-ray diffraction (b). Photographs of $H M P-T C N Q$ microcrystal fraction before (c) and after separation (d) were taken under an optical microscope, field of view $1 \mathrm{~mm}$ horizontally. AFM images (e) show a UHV co-deposited thin film of TMP-TCNQ.

The measurements were carried out at the P09 HAXPES endstation of PETRA III (Hamburg) at a photon energy of $6 \mathrm{keV}$. Due to the large inelastic mean free path of the fast electrons the probing depth is of the order of $10-15 \mathrm{~nm}$ for $5-6 \mathrm{keV}$ photoelectrons and thus surface contaminations play only a minor role. Photoelectrons with kinetic energies up to $15 \mathrm{keV}$ can be measured in different experimental geometries by means of a high-energy electron analyzer (type SPECS Phoibos 225 HV) with a combined Delayline Detector (type Surface Concept) [19]. For the present measurements the overall energy resolution (electrons and photons) was set to $440 \mathrm{meV}$. The beam was focused down to $0.2 \mathrm{~mm} \times 0.4 \mathrm{~mm}$ spot size. The spectra were recorded in normal emission geometry at nearly grazing photon incidence $\left(\approx 2^{\circ}\right)$.

\section{HAXPES spectra}

\subsection{Spectra of solution-grown co-crystals}

Oxygen is only contained in the methoxy groups of HMP and TMP. The oxygen spectra thus constitute a local spectroscopic probe of the functional group in the donor moieties. Fig. 2 shows core-level spectra of $\mathrm{O} 1 \mathrm{~s}$ for three different HMP-TCNQ crystal fractions ( $\mathrm{a}, \mathrm{b}$, c) and the corresponding spectrum of pure $H M P($ d). Fig. 3 displays the analogous results for two fractions of TMP-TCNQ. All spectra were analyzed by a multipeak least-squares fit routine, deconvoluting the spectra. The line shape was simulated by convoluting Gaussian and Lorentzian contributions (so-called Voigt profile). The Gaussian part is defined by the instrumental resolution (beamline + spectrometer) and has a full width at half maximum (FWHM) of $440 \mathrm{meV}$. The fit reveals three clearly distinguishable peaks $\mathrm{B}$, $\mathrm{C}$ and $\mathrm{D}$ for the compounds, in comparison with the single line $\mathrm{A}$ for pure donor material, spectra (d). Peak A is perfectly fitted by a Gaussian-Lorentzian profile with width $1.73 \mathrm{eV}(1.78 \mathrm{eV}) \mathrm{FWHM}$ for $H M P(T M P)$. Its centre is located at a binding energy of $533 \mathrm{eV}$ for HMP and for TMP and is used as zero-reference for the shift scale (top abscissa).

The 01 s spectra of the microcrystal fractions are substantially different from the single-line spectra of the pure donors. However, they have the same characteristic shape for HMP-TCNQ and 


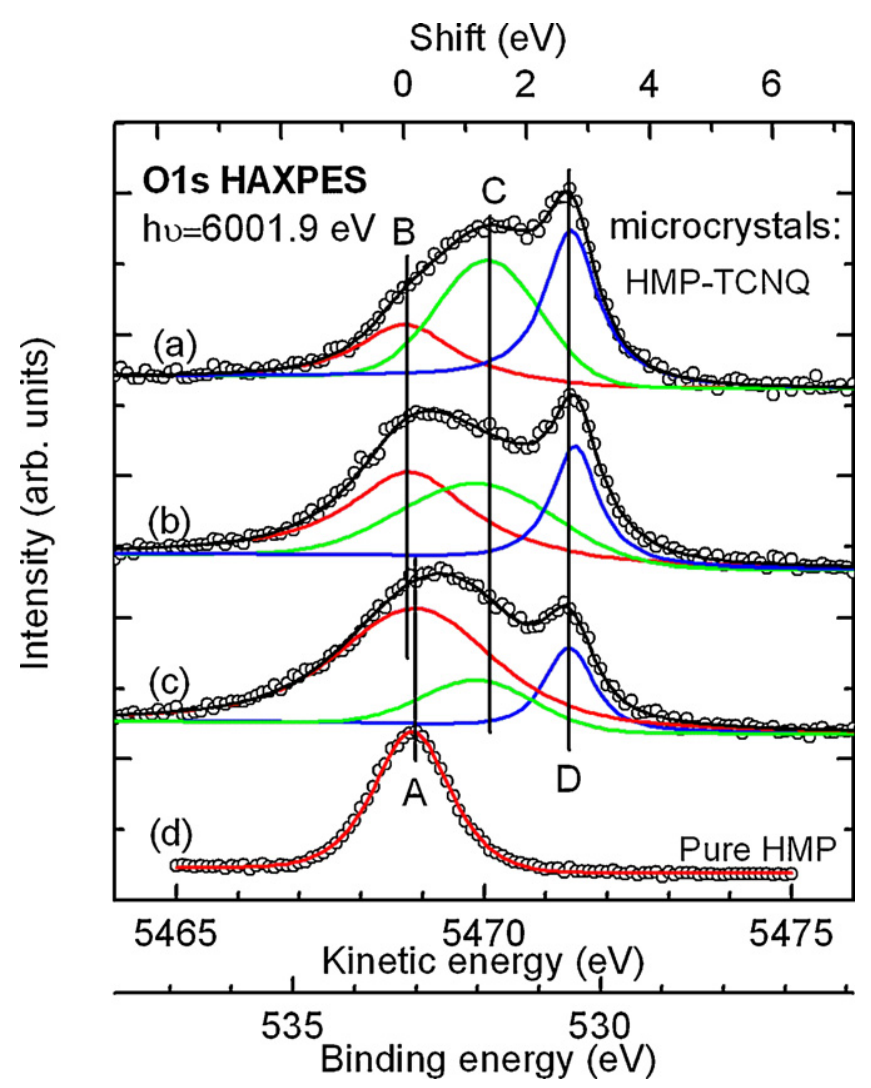

Fig. 2. O 1s HAXPES core-level spectra for three fractions of microcrystals of the CT complex HMP-TCNQ grown in solutions with different mixtures HMP:TCNQ $=1: 2$ (a), 1:1 (b) and 2:1 (c) together with the spectrum of the pure donor material HMP (d).

TMP-TCNQ. In the following values for TMP-TCNQ are given in brackets. The fits reveal one peak close to the position of $\mathrm{A}$ and two further peaks on the low-binding-energy side, being indicative of a change of the chemical environment of the HMP and TMP molecules. There are two main contributions to the level shifts. The formation of the complex changes the chemical environment of the molecules. In addition, the charge transfer itself leads to a further shift as will be discussed in Section 4. As signal B occurs almost at the same energy as peak $A$, we attribute it to the residue of small microcrystals of the pure donors in the separated fraction, as discussed in Fig. 1. Signal C is shifted by $1.4 \mathrm{eV}(0.9 \mathrm{eV})$ and signal D by $2.6 \mathrm{eV}(2.3 \mathrm{eV})$ from the reference line $\mathrm{A}$ of the pure donors. It is remarkable that peak $\mathrm{D}$ has a line width of only $1.15 \mathrm{eV}(0.8 \mathrm{eV})$ FWHM that is much smaller than the width of line $A$ of the pure donors. The CT phase is thus characterized by two peaks separated by about $1.2 \mathrm{eV}$.

The peak areas can be analyzed for a determination of the relative amounts of residues of pure donor material in the different samples (signal B) and of different chemical species in the CT phase (signals $\mathrm{C}$ and D). The HMP-TCNQ fraction in Fig. 2a was grown in an $H M P$-poor solution, fraction (b) in a solution with $1: 1$ stoichiometry and fraction (c) in an HMP-rich solution. From the ratios of the area of signal $B$ to the total integral of the $O 1 \mathrm{~s}$ signal $(B+C+D)$ we determine a contribution of $20 \%, 42 \%$ and $73 \%$ of pure HMP crystallites in fractions (a), (b) and (c), respectively. The ratios of the two peaks associated with the CT phase will be analyzed in Section 4 .

For TMP-TCNQ the abundances of residual pure donor material are considerably higher, see large signal $\mathrm{B}$ in Fig. 3a and b. Integration yields $B:(B+C+D)=61 \%$ and $63 \%$ for crystal fractions from the TMP-poor and TMP-rich solutions, respectively. The analysis of

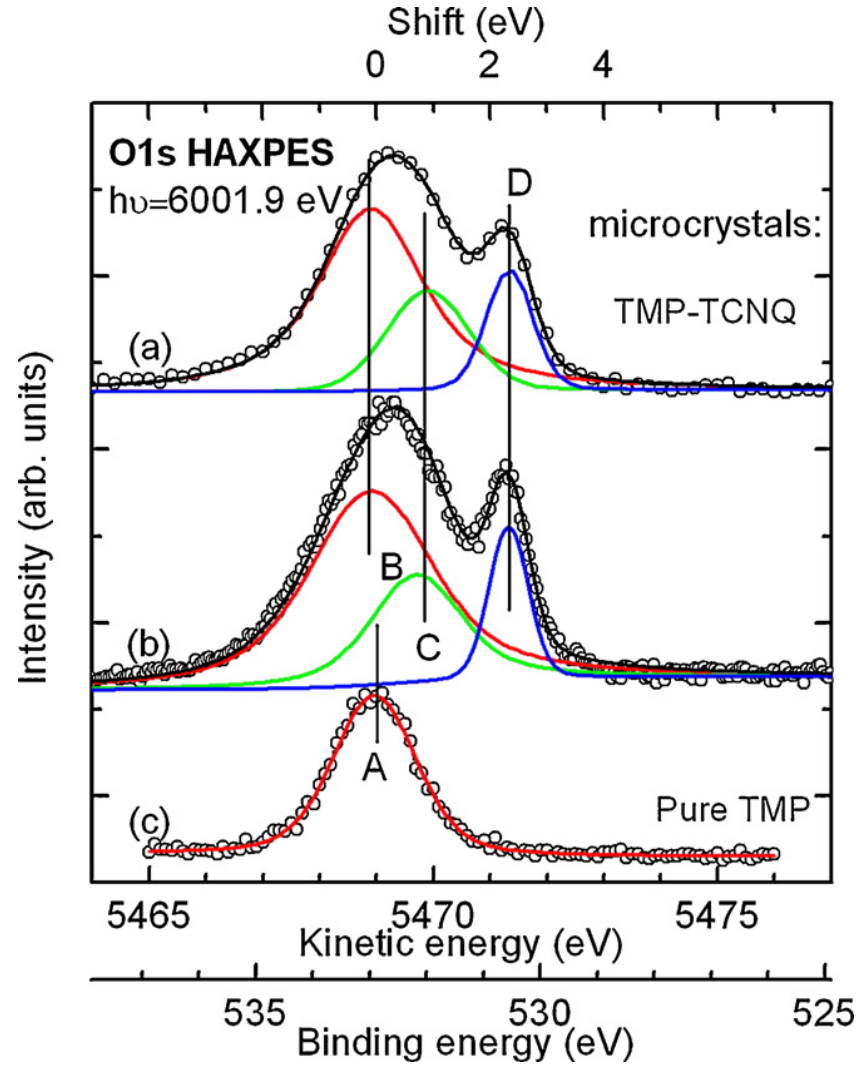

Fig. 3. O 1s HAXPES core-level spectra for fractions of microcrystals of the CT complex TMP-TCNQ grown in solutions with different mixtures TMP:TCNQ $=1: 2$ (a) and 2:1 (b) together with the spectrum of the pure donor material TMP (c).

the CT phase will be done in the same way as for HMP-TCNQ cf. Section 4.

Nitrogen is only contained in the cyano-group of TCNQ. The nitrogen spectra thus provide a local probe in the acceptor moiety. Fig. 4 shows $\mathrm{N}$ 1s spectra of the TMP-TCNQ complex and the corresponding spectrum of pure $T C N Q(\mathrm{c})$. The latter spectrum is well-known $[9,13,14,20]$, our spectrum agrees perfectly with the literature spectra. It shows a strong main peak E (width $0.95 \mathrm{eV}$ FWHM) and a weaker signal $\mathrm{F}$ located $2.6 \mathrm{eV}$ at higher binding energy. This weak signal is assigned as a highest occupied molecular orbital (HOMO)-lowest unoccupied molecular orbital (LUMO) shake-up satellite [21]. The shift scale (top abscissa) refers to the centre of the main line $\mathrm{E}$ of pure TCNQ at a binding energy of $398.5 \mathrm{eV}$.

The spectra of TMP-TCNQ (as well as HMP-TCNQ not shown) differ substantially from the spectrum of pure $T C N Q$ (c). The leading signal is the broad peak $\mathrm{H}$ (width $1.4 \mathrm{eVFWHM}$ ) that occurs at a shift of $0.9 \mathrm{eV}$ with respect to the main peak $\mathrm{E}$ of pure $T C N Q$. A smaller signal G occurs at the same position and has approximately the same width as signal E. One can conclude that this peak corresponds to a small admixture of pure TCNQ crystallites in the microcrystal fractions, as visible in the microscope images. A third signal I (width $1.5 \mathrm{eV}$ ) occurs at a shift of $2.0 \mathrm{eV}$ in the same direction. In this case the shift occurs towards higher binding energies, i.e. oppositely to the shift observed in the donor moiety. The intensity of signal I is too high to interpret it as a shake-up satellite of $\mathrm{H}$. We conclude that I also derives from the main line $\mathrm{E}$ but with a stronger chemical shift.

Similarly as for the oxygen spectrum the spectroscopic fingerprint of the CT phase consists of two shifted signals $\mathrm{H}$ and I, separated by $1.1 \mathrm{eV}$. The satellite at a loss of $2.6 \mathrm{eV}$ is missing. This can be understood because the HOMO-LUMO transition of pure $T C N Q$ is strongly altered upon formation of the complex. In fact, the 


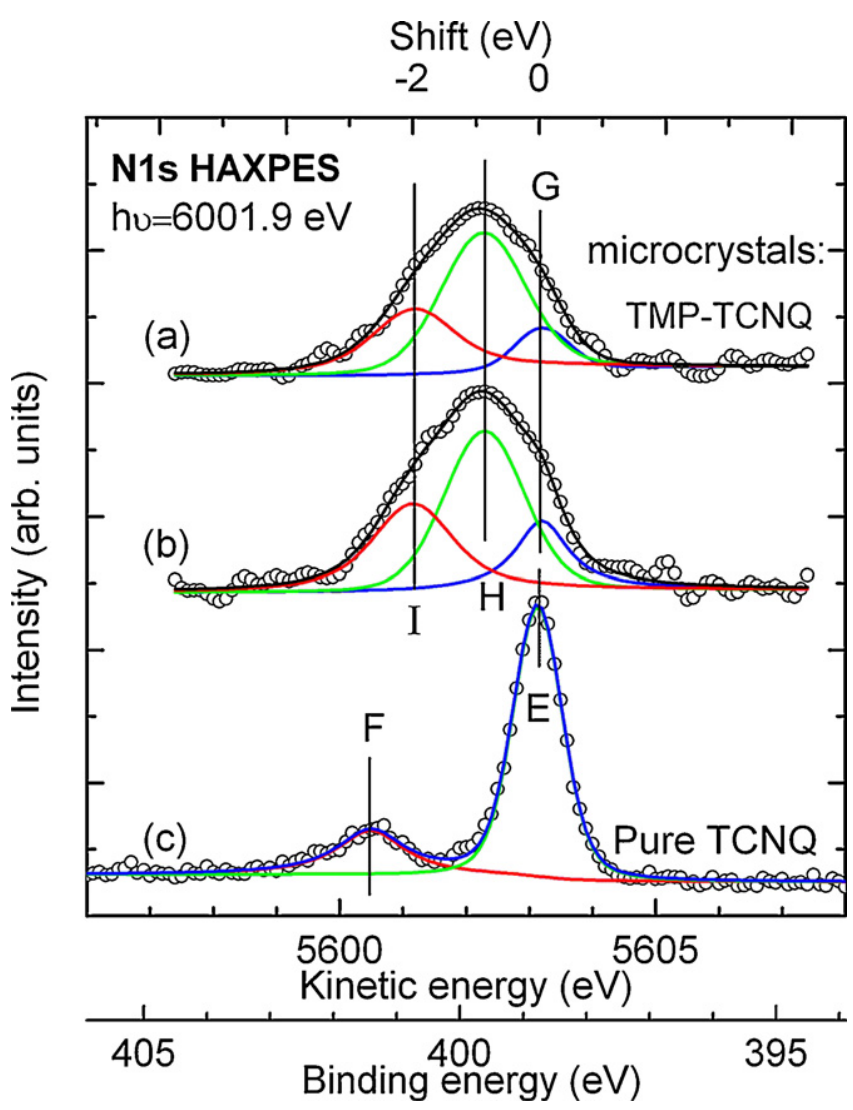

Fig. 4. N 1s HAXPES core level spectra for fractions of microcrystals of the CT complex TMP-TCNQ grown in solutions with different mixtures TMP:TCNQ $=1: 2$ (a) and $1: 1$ (b) together with the spectrum of the pure acceptor material TCNQ(c).

HOMO-LUMO gap of TMP-TCNQ is only $1.25 \mathrm{eV}$, as we have measured using scanning tunnelling spectroscopy [11]. HOMO-LUMO shake up in the complex would therefore lead to satellite signals shifted by $1.25 \mathrm{eV}$ towards higher binding energies. From the ratios of the area of signal $\mathrm{G}$ to the total integral of the $\mathrm{N} 1 \mathrm{~s}$ signal $(\mathrm{G}+\mathrm{H}+\mathrm{I})$ we determine a contribution of $11 \%$ and $19 \%$ of pure TCNQ crystallites in fractions (a) and (b), respectively. The degree of charge transfer will be compared with the evaluation of the $01 \mathrm{~s} \mathrm{spectra}$ for the TMP-TCNQ complex in Section 4.

The $\mathrm{N}$ 1s spectra gave clear evidence of radiation damage as shown in Fig. 5. An additional, strongly shifted signal (arrow) occurred at $3.5 \mathrm{eV}$ after about $30 \mathrm{~min}$ of continuous irradiation at the same position. A similar behaviour was observed for TMP-TCNQ (spectrum b) and HMP-TCNQ (spectrum c). Immediately after exposure spectrum (a) was measured, where this signal was absent. This behaviour was not observed in the 01 s spectra. Sample damage was accounted for by frequently changing the position of the X-ray beam on the sample. A similar tendency was observed for TTF-TCNQ films and bulk material by Ikemoto et al. [22]. The N 1s spectra showed a new peak at higher binding energy, but no $S$ 2p spectra. The authors suggest that there is a chemical reaction between adsorbed molecules and $T C N Q^{-}$which is enhanced in an unknown way by X-ray irradiation. In the present case, we can exclude this possibility due to the bulk sensitivity, connected with a low contribution of surface contaminations in the HAXPES spectra. This points on radiation damage in the bulk due to e.g. bond breaking.

\subsection{Spectra of UHV co-deposited thin films}

The thin-film samples were deposited immediately before the HAXPES experiment. They were measured under the same settings

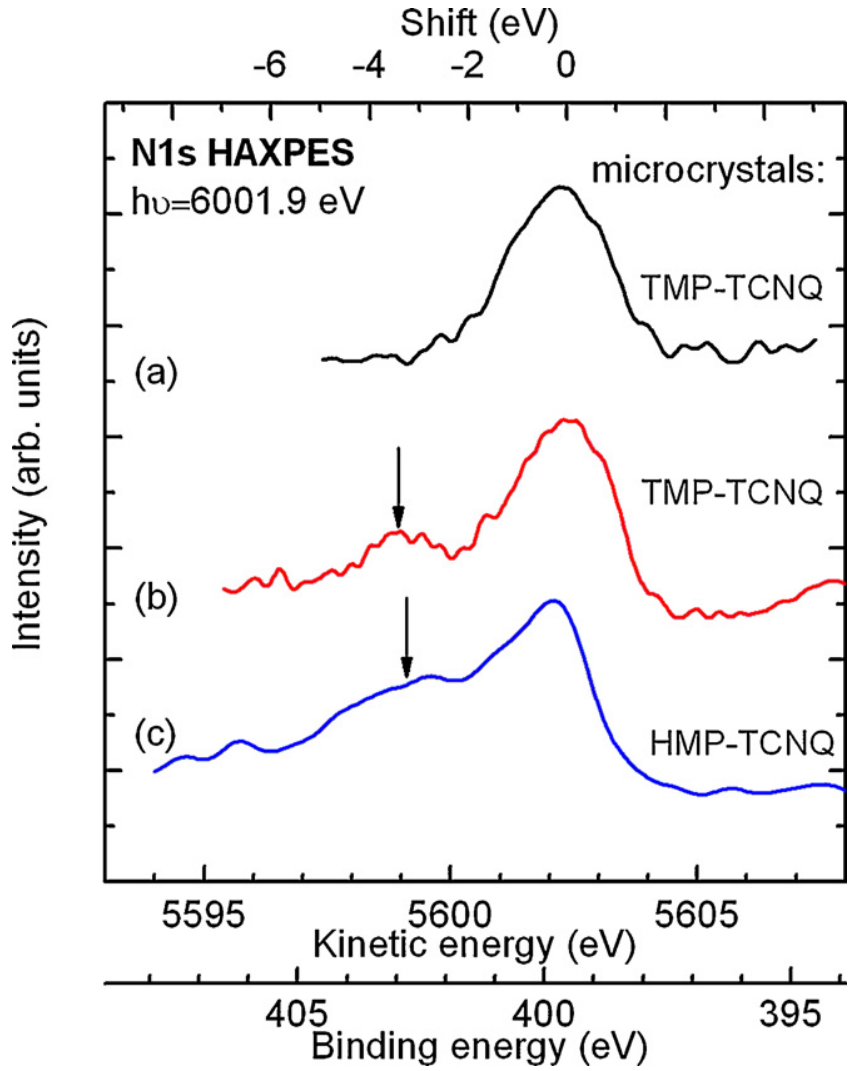

Fig. 5. Evidence of radiation damage in N 1s HAXPES core level spectra. Immediately after exposure spectrum (a) is measured, whereas after about 30 min of continuous irradiation at the same position an additional signal occurs, marked by arrows for TMP-TCNQ (b) and HMP-TCNQ (c).

as the microcrystalline samples. Fig. 6 shows the 0 1s core-level spectrum of the TMP-TCNQ complex (a) and the corresponding spectrum of pure TMP (b). The single peak A of the TMP spectrum with its centre at a binding energy of $533 \mathrm{eV}$ serves as zeroreference for the shift scale.

The spectrum of the complex is deconvoluted into three signals B, C and D. Signal B lies only $0.5 \mathrm{eV}$ off the position of peak A from the pure donor. As in the case of the solution-grown crystals, signal $B$ can be attributed to the formation of pure donor inclusions or islands in the film. From the ratio of the area of signal B to the sum of areas B, C and D we determine a contribution of $26 \%$ of pure TMP inclusions or islands in the thin film. The shifts of peaks $\mathrm{C}(1.87 \mathrm{eV}$ FWHM) and D (1.26 eV FWHM) are $2.47 \mathrm{eV}$ and $3.67 \mathrm{eV}$, respectively. These values are significantly larger than those observed for the case of the microcrystal fractions, shown in Figs. 2 and 3. The direction of the shifts is the same. The larger shift may indicate a larger change of chemical environment of the TMP molecules due to the charge transfer in the complex on the surface.

\section{Discussion}

It is eye-catching in all 0 1s spectra (Figs. 2, 3 and 6) that the structure changes from the single line of the pure donors HMP and TMP to a spectrum with three distinct peaks B, C and D for the microcrystal fractions and thin film of the complexes. The spectral weight is strongly shifted towards higher kinetic energy, i.e. lower binding energy (which rules out charging as origin of these shifts). Signal B is indicative of residues of pure donor material with abundances between $20 \%$ (for the HMP-poor solution) and $67 \%$ (for the HMP-rich solution) in the microcrystal fractions and 26\% in the UHV-deposited film (Fig. 6). Optical microscopy revealed 


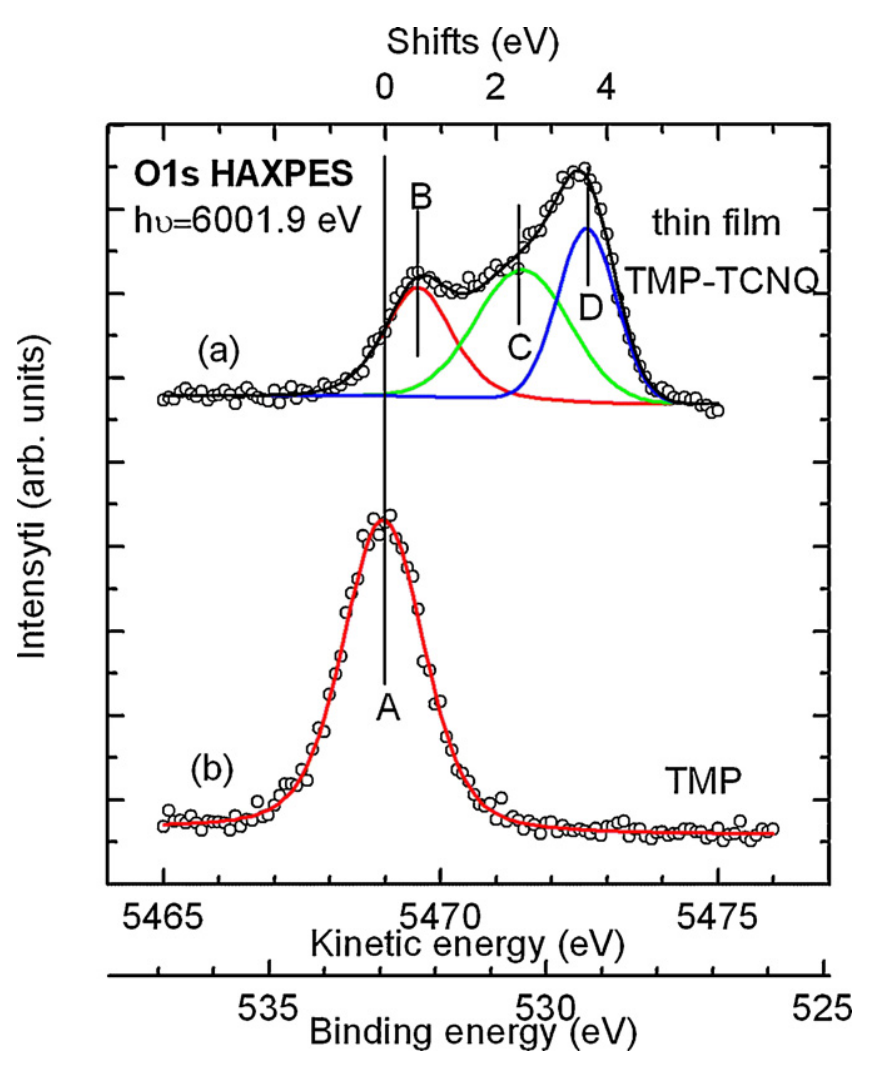

Fig. 6. O 1s HAXPES spectra for a co-deposited thin film of the CT complex $T M P-T C N Q$ grown in UHV (a), together with the spectrum of the pure donor material $T M P(b)$.

residues of small transparent (donor) crystallites in the microcrystal fractions that could not be removed via mechanical separation because they were too small. The co-deposited film obviously contains inclusions of the pure donor as well. A possible explanation may be that the composition of the deposited molecular beam deviated from 1:1 stoichiometry or the sticking coefficient of TMP is higher than that of $T C N Q$, so that an excess of donor molecules existed in the deposited material. It can be assumed that these inclusions are small and probably embedded into the matrix of the CT complex. This may explain the small energetic shift of peak B in Fig. 6.

Before we discuss the signals associated with the CT phase, $C$ and $D$, we first consider the action of a charge transfer on the core-level positions in the donor and acceptor moieties. The charge transfer from donor to acceptor shifts the core-level positions as sketched in the simplified energy scheme shown in Fig. 7. The model takes into account the screening of the Coulomb attraction of the nucleus by the different number of electrons in the valence shell. This core-level screening increases with increasing number of valence electrons and leads to a reduction of the binding energy. For the donors, probed by $\mathrm{O} 1 \mathrm{~s}$ photoemission, we schematically sketch the situation of a completely filled HOMO of the methoxy group (symmetry $2 e$ for an isolated methoxy group) (Fig. 7a) and one vacancy in this orbital (b). It has been found for the surface methoxy species on copper that this orbital (being the HOMO of a fully anionic methoxy group) plays an important role for the charge transfer [23]. We thus expect a shift of the donor core levels to higher binding energies when the donor orbitals loose electron charge or when the donor is fully ionized as sketched in the figure. This suggests that the signal at lowest binding energy (signal D in Figs. 2, 3 and 6) corresponds to the neutral donor molecule $H M P^{0}$ or TMP ${ }^{0}$ in the complex and the signal at higher binding energy (signal $\mathrm{C}$ ) to the charged donor $H M P^{+}$or $T M P^{+}$ (a)

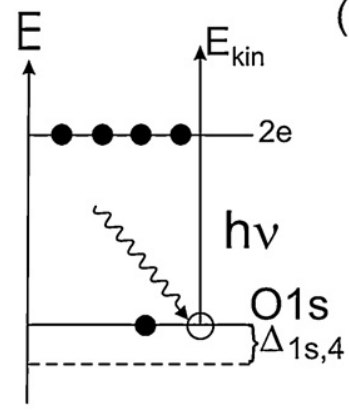

(c)

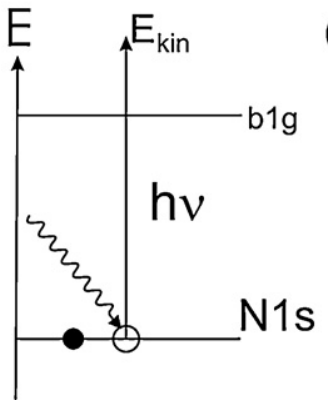

(b)

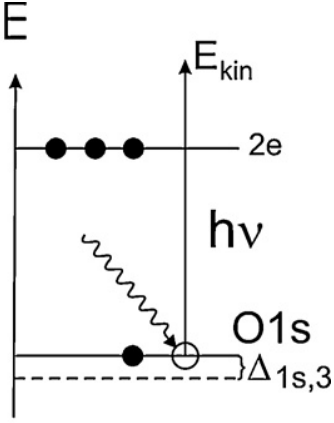

(d)

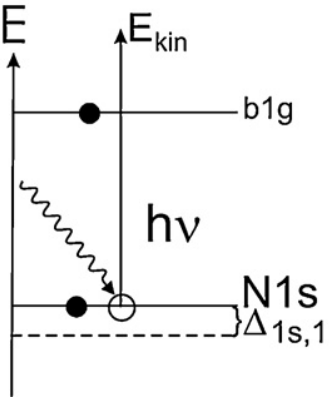

Fig. 7. Energy scheme of the core-hole screening effect in the photoemission transitions observed.

For the acceptor, probed by $\mathrm{N} 1 \mathrm{~s}$ photoemission, the situation is reversed as depicted in Fig. 7c and d. The charge transfer delivers an additional electron, here in the (formerly empty) $b_{1 g}$ orbital located essentially at the cyano group. This orbital is the LUMO of the cyano group [24]. This electron lowers the binding energy via additional core-hole screening. The acceptor core levels are thus shifted to lower binding energies when the acceptor gains electron charge or when it is fully ionized. This means that signal I (at higher binding energy) corresponds to the neutral acceptor molecule $T C N Q^{0}$, whereas signal $\mathrm{H}$ corresponds to the charged acceptor $\mathrm{TCNQ}^{-}$.

This simple model just takes into account a charge loss or excess in the valence orbitals. It thus covers only the aspect of the relative order of the core-level signals $\mathrm{C}$ and $\mathrm{D}(\mathrm{O} 1 \mathrm{~s})$ and $\mathrm{H}$ and $\mathrm{I}(\mathrm{N} 1 \mathrm{~s})$ associated with the CT complex. Further contributions to the total chemical shift arise due to changes, e.g. in the electrostatic environment of the emitter atom. We discussed in context of Fig. $1 \mathrm{~b}$ that many short contacts between atoms of neighbouring molecules are found in average with $0.32-0.35 \mathrm{~nm}$, giving rise to a substantial contribution in the chemical shift. In addition, we know from the X-ray diffraction experiments that some of the methoxy groups undergo a conformational transition. This is another contribution to the total change in the nearest chemical surrounding that the oxygen atoms experience. It will cause different shifts for different methoxy groups, leading to a broadening of the core-level signals (which represent the weighted superposition of all conformations).

The spectra indeed reveal the expected $\mathrm{O} 1 \mathrm{~s}$ spitting between neutral and charged donors. The distance of signals $C$ and $D$ is $1.2-1.4 \mathrm{eV}$ for the microcrystals and $1.2 \mathrm{eV}$ for the TMP-TCNQ film. The width of signal $C$ is rather large, whereas signal $D(0.9-1.2 \mathrm{eV}$ FWHM) is even narrower than the single line A in pure HMP or TMP (1.6-2.0 eV FWHM). The N 1s splitting between neutral and charged acceptors (distance of signals I and $\mathrm{H}$ in Fig. 4 ) is $1.1 \mathrm{eV}$, close to the value found for TMTSF-TCNQ [10].

For TTF-TCNQ and related compounds a splitting of about $1.5 \mathrm{eV}$ was observed in the $S 2 p(N$ 1s) peak positions between the neutral molecules $T T F^{0}\left(T C N Q^{0}\right)$ and ionic $T T F^{+}\left(T C N Q^{-}\right)$[1,8,20]. Analogously, an evaluation of the molecular mixed valence of TCNQ was made for the complex BTV [bis(ethylenedithio)tetrathiafulvalene], 
$M(d m i t){ }_{2}$ (dmit=1,3-dithio-2-thione-4,5-dithiolate) VinyloguesTCNQ. A binding-energy difference of $1.6 \mathrm{eV}$ was measured between the neutral and ionic acceptor molecule [9]. Peaks G and $\mathrm{H}$ occur at similar positions as the signals observed in the spectrum of a $\mathrm{Cu}-\mathrm{TCNQ}_{2}$ complex [21]. These authors discuss the term "incomplete charge transfer" and state that is does not mean a partial electron transfer or sharing of electron density between TCNQ and the donor species but a complete electron transfer to only some TCNQ moieties [20]. Grobman et al. conclude that the N 1s XPS spectrum of TTF-TCNQ is strong evidence for partial charge transfer in the crystal, with between 1.3 and $2 T_{C N Q^{-}}$molecules for each neutral molecule, on the time scale of XPS [8].

In our previous work on K-edge NEXAFS for the same samples [25] we have observed shifts between the resonance positions for the pure donor and acceptor moieties and the compound of about $0.5 \mathrm{eV}$ for TMP-TCNQ and $0.75 \mathrm{eV}$ for HMP-TCNQ. These shifts are substantially smaller than the shift in the HAXPES spectra of the present paper. An earlier UPS study revealed shifts of the HOMO of about $0.8 \mathrm{eV}$ for TMP-TCNQ [11]. These differences reflect the different information content in core-level and valence-level photoemission and X-ray absorption. UPS reflects a shift in the valence bands of the compounds which is a consequence of the hybridization of valence orbitals of donor and acceptor forming new energy bands in the solid. In addition, UPS probes only the surface and could thus be influenced by a possible reduced charge transfer in the topmost layers. In NEXAFS we observe transitions from the core-level to an empty $\pi$ or $\sigma$-state in the valence region. NEXAFS resonances do not reflect the energy of a hole-state but rather a resonant transition in the complex.

The behaviour for NEXAFS resonance positions is fundamentally different from the situation for photoemission as discussed in Fig. 7. In addition to the initial state effect caused by the screening of the nucleus, the final state is pulled down by the presence of the core hole. The amount of final state shift is the larger, the smaller the total occupation of the final-state orbital. The essential point is that this lowering of the final-state energy levels counteracts the lifting of the initial levels so that both contributions may cancel each other to a certain extent. As a consequence, the shifts observed in NEXAFS are expected to be smaller than those observed in HAXPES, confirming the experimental results.

From the relative HAXPES intensities of signals $C$ and $D$ in the oxygen spectra we might estimate the degree of charge transfer. Based on the assumption that signal B reflects pure donor inclusions, the total signal of the complex is the sum $\mathrm{C}+\mathrm{D}$. For HMP-TCNQ the ratio of peak $\mathrm{C}$ to this total intensity is $C:(C+D)=0.64$ for fraction (a), 0.63 for fraction (b) and 0.51 for fraction (c) in Fig. 2. For TMP-TCNQ the fit yields a ratio of the peak integrals of $C:(C+D)=0.65$ for fraction (a) and 0.66 for fraction (b) in Fig. 3 and 0.61 for the thin-film sample, Fig. 6 . We estimate an error of about 0.15 for these values. By evaluation of the relative intensities of signals $\mathrm{H}$ and $\mathrm{I}$ in the $\mathrm{N} 1 \mathrm{~s}$ spectra we find the corresponding ratios of 0.64 for fraction (b) and 0.65 for fraction (c) in Fig. 4. Here we estimate an error of 0.25 due to the large widths of both peaks and higher noise in the nitrogen spectra. Obviously all these ratios are the same within the experimental error limits. This indicates that the degree of charge transfer is similar in all samples, although the admixture of donors and acceptors in the solution differed by up to a factor of 4 .

\section{Summary and conclusion}

In this work we have performed a hard X-ray photoemission experiment on a new class of CT compounds based on a functionalized polycyclic aromatic hydrocarbons as donors in complexes with the classical electron acceptor tetracyanoquinodimethane TCNQ. The donors are based on the parent molecule pyrene with four or six methoxy-groups at the periphery (tetra- and hexamethoxypyrene TMP and HMP). These groups donate charge to the $\pi$-electron system. The CT complex was either co-crystallized from solution by vapour diffusion or co-deposited by thermal evaporation in ultrahigh vacuum. Owing to the large information depth of $15-20 \mathrm{~nm}$ surface impurities from the solvent or ambient air were not significant. For comparison, crystalline and thin-film samples of pure donor and acceptor material have been studied under identical conditions.

As oxygen is only contained in the functional group of the donors, O 1s HAXPES provides a local probe in the methoxy group. Likewise, nitrogen is contained only in the cyano-group of TCNQ and $\mathrm{N} 1 \mathrm{~s}$ spectra gives access to the acceptor sites. Both the $\mathrm{O} 1 \mathrm{~s}$ and $\mathrm{N} 1 \mathrm{~s}$ spectra give evidence of two new energy levels in the CT complex that are shifted with respect to the single $\mathrm{O} 1 \mathrm{~s}$ line of pure HMP (TMP) or the leading line of $\mathrm{N} 1 \mathrm{~s}$ in $T C N Q$. The oxygen lines are shifted by $1.4 \mathrm{eV}$ and $2.6 \mathrm{eV}$ for HMP-TCNQ and by $0.9 \mathrm{eV}$ and $2.3 \mathrm{eV}$ for TMP-TCNQ towards lower binding energies and the nitrogen lines by $0.9 \mathrm{eV}$ and $2.0 \mathrm{eV}$ towards higher binding energies in TMP-TCNQ. A non-shifted $\mathrm{O} 1 \mathrm{~s}(\mathrm{~N} 1 \mathrm{~s})$-signal originates from residues of pure donor (acceptor) crystallites in the sample fraction, which were also visible under the optical microscope. We interpret the two new levels as fingerprints of the neutral and ionic species in the complex. For the donor the loss of charge leads to a reduced core-hole screening thereby increasing the core-level binding energies for the ionic state. For the acceptor the gain in charge in the valence region leads to an increased screening, lowering the corelevel binding energies in the ionic state. The $\mathrm{N} 1 \mathrm{~s}$ level shift between neutral and ionic TCNQ of about $1.1 \mathrm{eV}$ in TMP-TCNQ is in reasonable agreement with the literature value of $1.4 \mathrm{eV}$ found for TTF-TCNQ [8]. The $O 1 \mathrm{~s}$ level shift of $1.2-1.4 \mathrm{eV}$ between neutral and ionic HMP or TMP in the complex is somewhat smaller than the value of $1.6 \mathrm{eV}$ found for $\mathrm{S} 2 \mathrm{p}$ in the donor tetrathiafulvalene TTF in TTF-TCNQ [1].

UPS spectra [11] and the NEXAFS resonances [25] show smaller energetic shifts $(0.5-0.8 \mathrm{eV})$, in opposite directions for the donor and acceptor molecules. The present shifts of $\mathrm{N} 1 \mathrm{~s}$ and $\mathrm{O} 1 \mathrm{~s}$ are a factor of 3-5 larger than the shifts in the K-edge NEXAFS spectra for the same samples.

From the ratio of the corresponding signal intensities (peak areas) we derive degrees of charge transfer between 0.51 and 0.65 . These values lie close to the charge transfer of $2 / 3$ that was found in previous work by Pouget et al. for the $N$-methyl-2pyrrolidone (NMP)-TCNQ CT complex. A charge transfer of $2 / 3$ can be explained by the disorder of methyl groups of NMP-TCNQ. This effect was observed by diffuse X-ray scattering and conductivity measurements $[26,27]$. These results correlate very well with our deductions concerning a redistribution of the charge in $H M P(T M P)$ $T C N Q$ complexes. One can conclude that the arrangement of the donors and acceptors in the studied CT crystals is similar to the well know classical NMP-TCNQ system and maybe connected with the randomness in the methoxy group location.

In conclusion, the bulk-sensitive photoemission technique HAXPES gives access to the degree of charge transfer in solution-grown organic donor-acceptor co-crystals. The surfaces of such compounds cannot be prepared using common preparation tools like ion bombardment. The large information depth of $15-20 \mathrm{~nm}$ eliminates the problem of surface contamination completely. For the family of $H M P(T M P)-T C N Q$ complexes the degree of charge transfer was determined from the relative intensities of the neutral and ionic signals. $\mathrm{O} 1 \mathrm{~s}$ and $\mathrm{N} 1 \mathrm{~s}$ spectra give direct access to the functional groups of the donors and acceptors, respectively. In the future, the same technique can be applied to determine the charge transfer in larger disc-shaped polycyclic aromatic hydrocarbons like coronene-derivatives hexamethoxycoronene (donor) and coronene-hexaone (acceptor) which were synthesized recently [28] 
or to even larger members of this family of nanographenes. The specific tailoring of donor- or acceptor character can be utilized for designing new CT complexes with extended $\pi$-electron systems of the constituents.

\section{Acknowledgements}

The project is funded through Transregio SFB TR 49 (Frankfurt, Mainz, Kaiserslautern), Graduate School of Excellence MAINZ and Centre for Complex Materials (COMATT), Mainz. We especially wish to thank H.J. Elmers and J.P. Pouget for fruitful discussion, S. Naghavi for helping with theoretical understanding of the charge transfer effect in the complexes, and W. Drube with team for support during measurements at beamline P09 (PETRA III).

\section{References}

[1] I. Ikemoto, T. Sugano, H. Kuroda, Chem. Phys. Lett. 49 (1977) 45-48.

[2] J.M. Williams, J.R. Ferraro, R.J. Thorn, K.D. Karlson, U. Geiser, H.H. Whang, A.M. Kini, M.H. Whangbo, Organic Superconductors (including Fullerenes) Synthesis, Structure, Properties and Theory, Prentice-Hall, New York, 1992.

[3] H.S. Nalwa, Handbook of Organic Conductive Molecular and Polymers, Wiley, 1997.

[4] K. Müllen, J.P. Rabe, Acc. Chem. Res. 41 (2008) 511.

[5] M.D. Watson, A. Fechtenkötter, K. Müllen, Chem. Rev. 101 (2001) 1267.

[6] M. Randic, Chem. Rev. 103 (2003) 3449.

[7] J. Wu, W. Pisula, K. Müllen, Chem. Rev. 107 (2007) 718-747.

[8] W.D. Grobman, B.D. Silverman, Solid State Commun. 19 (1976) 319-322.

[9] L. Yu, D. Zhu, Physica C 282-287 (1997) 1899-1900.

[10] I. Ikemoto, K. Kikuchi, K. Yakushi, H. Kuroda, K. Kobayashi, Solid State Commun. 42 (1982) 257-259.
[11] K. Medjanik, S. Perkert, S. Naghavi, M. Rudloff, V. Solovyeva, D. Chercka, M. Huth, S.A. Nepijko, T. Methfessel, C. Felser, M. Baumgarten, K. Müllen, H.J. Elmers, G. Schönhense, Phys. Rev. B 82 (2010) 245419.

[12] S.-i. Kawano, M. Baumgarten, D. Chercka, V. Enkelmann, K. Müllen, Chem. Eur. J., submitted for publication.

[13] J.M. Lindquist, J.C. Hemminger, J. Phys. Chem. 92 (1988) 1394-1396.

[14] J. Giergiel, S. Wells, T.A. Land, J.C. Hemminger, Surface Sci. 255 (1991) $31-40$

15] Yu.G. Borodko, M.G. Kaplunov, T.M. Moravskaya, K.L. Pokhodnya, Phys. Stat Sol. 83 (1977) 141.

[16] K. Medjanik, D. Kutnyakhov, S.A. Nepijko, G. Schönhense, S. Naghavi, V. Alijani, C. Felser, N. Koch, R. Rieger, M. Baumgarten, K. Müllen, Phys. Chem. Chem. Phys. 12 (2010) 7184-7193.

[17] I.N. Stranski, L. Von Krastanow, Abhandlungen der MathematischNaturwissenschaftlichen Klasse, Akademie Wissenschaften Literatur Mainz 146 (1939) 797.

[18] V. Solovyeva, K. Keller, M. Huth, Thin Solid Films 517 (2009) 6671.

[19] A. Gloskovskii, G. Stryganyuk, G.H. Fecher, C. Felser, S. Thiess, H. Schulz-Ritter, W. Drube, G. Berner, M. Sing, R. Claessen, M. Yamamoto, JESRP, in press.

[20] W.D. Grobman, R.A. Pollak, D.E. Eastman, E.T. Maas Jr., B.A. Scott, Phys. Rev. Lett 32 (1974) 534-537.

[21] J.M. Lindquist, J.C. Hemminger, Chem. Mater. 1 (1989) 72-78.

[22] I. Ikemoto, M. Yamada, T. Sugano, H. Kuroda, Bull. Chem. Soc. Jpn. 53 (1980) 1871-1876.

[23] K. Amemiya, Y. Kitajima, Y. Yonamoto, S. Terada, H. Tsukabayashi, T. Yokoyama, T. Ohta, Phys. Rev. B 59 (1999) 2307.

[24] J. Fraxedas, Y.J. Lee, I. Jimenez, R. Gago, R.M. Nieminen, P. Ordejon, E. Canadell, Phys. Rev. B 68 (2003) 195115.

[25] K. Medjanik, S.A. Nepijko, H.J. Elmers, G. Schönhense, P. Nagel, M. Merz, S. Schuppler, D. Chercka, M. Baumgarten, K. Müllen, H. Jeschke, R. Valenti, JACS, in press.

[26] J.P. Pouget, S. Megtert, R. Comes, A.J. Epstein, Phys. Rev. B 21 (1980) 486.

[27] J.P. Pouget, Z. Kristallogr. 219 (2004) 711-718.

[28] R. Rieger, M. Kastler, V. Enkelmann, K. Müllen, Chem. Eur. J. 14 (2008) 6322. 